\title{
Open complex distal femur intraarticular fracture with Hoffa extension, extensor mechanism tear and post-recovery secondary traumatic peri implant fracture: report on an uncommon chronology
}

\author{
Lavindra Tomar, Gaurav Govil*, Pawan Dhawan
}

Department of Orthopaedics, Max Super Speciality Hospital, Patparganj, Delhi, India

Received: 29 August 2020

Accepted: 07 October 2020

*Correspondence:

Dr. Gaurav Govil,

E-mail: gauravgovil@yahoo.co.in

Copyright: $@$ the author(s), publisher and licensee Medip Academy. This is an open-access article distributed under the terms of the Creative Commons Attribution Non-Commercial License, which permits unrestricted non-commercial use, distribution, and reproduction in any medium, provided the original work is properly cited.

\begin{abstract}
Complex distal femur fractures with Hoffa's extension are uncommon. An associated open fracture with extensor mechanism disruption are rarely reported. A secondary post-traumatic post-recovery peri-implant fracture is a rare presentation. 28-year-old pilon rider male sustained open complex fracture of left metaphyseal distal femur with Hoffa extension and tear in extensor mechanism. He was treated within a golden hour period of wound management. Primary stabilization by distal femoral locking plate and cannulated cancellous screw was done along with primary bone grafting and repair of extensor mechanism. At sixteen weeks, clinic-radiological progression allowed him his pre-injury activities. At seven months, a second trauma resulted in a non-prosthetic peri-implant femoral fracture, another unusual occurrence. Re-osteosynthesis with grafting was done. At twenty months of the primary injury, there was good functional outcome with a painless range of knee flexion to $130^{\circ}$ without any extensor lag. Open complex distal femur injury with an associated secondary hit peri-implant fracture presented with significant challenges in management of soft-tissue and reconstruction of bony defect. The early presentation within a golden hour period allowed an early injury assessment to implement primary biological fixation as a treatment modification. Aim was to achieve favorable functional outcome which allowed early return to pre-injury status.
\end{abstract}

Keywords: Distal femur fracture, Hoffa fracture, Open fracture, Peri implant fracture, Non-prosthetic femur fracture, Distal femoral locking plate

\section{INTRODUCTION}

Distal femoral fractures account for less than $1 \%$ of all fractures and comprise $4-6 \%$ of all femoral fractures. ${ }^{1,2}$ Open fractures comprise approximately $10 \%$ of the distal femoral fractures. ${ }^{3,4}$ Intra-articular distal femur fractures are high energy injuries with a rare coronal plane orientation of the fracture line. ${ }^{1-5}$ Hoffa's fracture may involve either of the femoral condyle but is more common on the lateral side because of natural knee valgus orientation. ${ }^{6,7}$ Open fractures with associated injuries of tibial spine, extensor tendon rupture have been reported rarely. ${ }^{5-8}$ Functional outcomes are compromised commonly due to infection, stiffness, nonunion and delayed rehabilitation.

A peri-implant fracture along distal femur locking plate has an incidence of 2.5-4.5\%. ${ }^{9}$ Factors commonly attributed for peri-implant fracture include rheumatoid arthritis, abnormal stress concentration at the plate construct end and osteoporosis especially in elderlies. However, open fractures do not affect the late complication of peri-implant fracture. ${ }^{9}$

We report a case of 28-year old pilon rider male who sustained a road traffic injury. The left knee had Gustilo- 
anderson type-III B, Arbeit gemeinschaft fur Osteosynthesefragen/Orthopaedic Trauma Association (AO/OTA) type 33-C3 fracture of the distal femur. ${ }^{10,11}$ Hoffa fracture and extensor mechanism disruption were associated injuries. Soft tissue management, fracture stabilization and reconstruction of bony defect posed challenges. Post recovery a second traumatic road traffic accident resulted in non-prosthetic peri-implant fracture which was managed with re-osteosynthesis and grafting.

\section{CASE REPORT}

A 28-year old male was admitted to casualty after a motorcycle accident sustaining injury around three hours back in his native village. Pilon rider on a two-wheeler, his flexed left knee hit hard against another two-wheeler with subsequent violent fall. First aid management with local wound repair along left knee was done at a primary health care centre. At our tertiary level care centre, trauma protocol assessment was done. There was no associated trauma to head, chest, abdomen and spine. Left lower limb revealed a painful, swollen knee joint with a loosely repaired lacerated wounds (Gustilo-Anderson type-II) on anteromedial aspect of the lower thigh and knee (figure 1a). Abnormal mobility of left lower thigh was present. No sign of acute ischemia of lower limb or any neurological deficit were present. Radiographic evaluation with anteroposterior and lateral views revealed comminuted fracture of distal femur with Hoffa extension (figure $1 \mathrm{~b}$ and 1c).

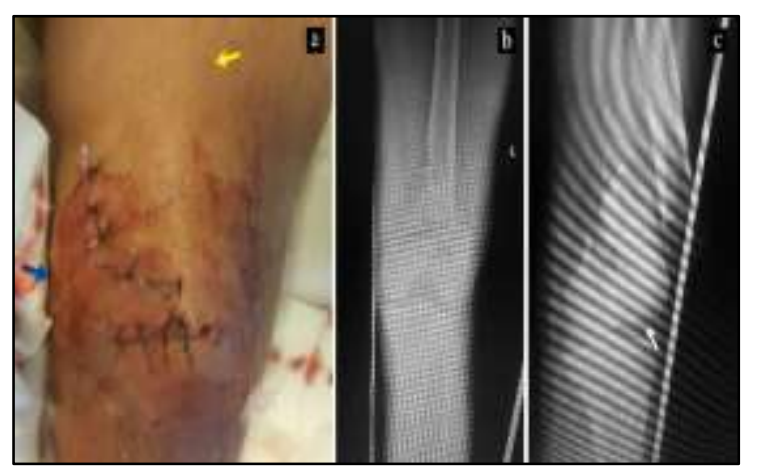

Figure 1: Clinical photograph (1a) of repaired wound thigh (yellow arrow) and knee (blue arrow on medial side) with preoperative plain anteroposterior (1b) and lateral (1c) radiographs of the fracture with white arrow at Hoffa's fragment.

An early surgical management was initiated within a golden hour period. The fractures were approached by connecting the open wounds over the anteromedial surface of the knee (figure 2a). The exposure was anterior midline approach with medial parapatellar retraction. The exposure was extended laterally to facilitate a direct exposure to the distal femur fracture. Rent in extensor mechanism was identified. Reassessment of wound after exploration confirmed a more severe grade of compounding (Gustilo-Anderson type-III B) (figure 2b). A thorough debridement of the wound was performed with copious saline irrigation. Reevaluation of fracture revealed a Hoffa fracture with articular cartilage involvement with severe metaphyseal supracondylar femoral comminution with loss of metaphyseal bony fragments (figure $2 \mathrm{c}$ ). No associated meniscal or cruciate injury noted. With the knee kept flexed at 90 degrees, approach gave direct exposure to the Hoffa fragment for an anatomical alignment. Temporary stabilization using $\mathrm{k}$-wires was done (figure 2d). Final fixation with antero-posteriorly directed partially threaded cannulated cancellous screw one each for both condyles was done with the screw heads buried under the articular cartilage (figure 3a). A temporary external fixator was an option however a biological primary fixation with a bridging AO locked distal femoral plate fixation ensured distal femur stabilization (figure $3 b$ ). Primary bone grafting was done with ipsilateral autologous iliac crest cortico-cancellous graft for defect reconstruction. Patella and patellar tendon were intact. Meticulous repair of quadriceps rent using nonabsorbable polypropylene sutures was done. Extensor retinaculum was repaired using polyglactin absorbable suture. Wound was closed over a suction drain (figure $3 \mathrm{c}$ ).

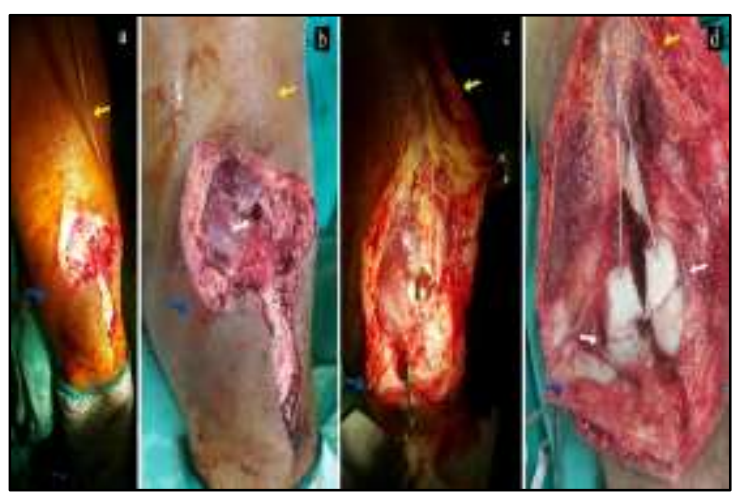

Figure 2: Per-operative images of thigh wound (yellow arrow), knee region (blue arrow on medial side) on suture removal (2a), showed rent (white arrow) in extensor mechanism (2b), after wound debridement

(2c), and Hoffa fracture (white arrow) pattern revealed temporarily stabilized $(2 d)$.

Stability of the fracture was considered satisfactory. A long plaster cast was applied post-operatively for three weeks. Wound healing was uneventful (figure 4a) and at three weeks a hinged knee brace was applied with progressive increase in passive range of knee movements. The patient was kept non-weight bearing for eight weeks and a monthly radiological assessment was done. With evidence of fracture consolidation, progressive full weight bearing was allowed. At four-month follow up, sound union was noted (figure $4 \mathrm{~b}$ and $4 \mathrm{c}$ ). A good clinical and functional recovery achieved with knee range of motion from 5 to 120 degrees with painless weight bearing.

At seven months after primary trauma, a second injury was sustained in another road traffic accident. A two-wheeler hit him while walking on the road from side reinjuring the left thigh. Radiographs revealed a femoral peri implant 
fracture occurring proximal to the distal femoral locking plate (figure $5 \mathrm{a}$ and $5 \mathrm{~b}$ ). The peri-implant fracture management posed multiple challenges including use of previous incision trajectory, increased risk of infection and alternate fixation techniques to be considered for an implanted distal femur plate. Previous healed incision was utilized to expose the fracture. Re-osteosynthesis with interfragmentary screw fixation of femoral shaft and a longer AO locked distal femur bridge plate fixation supplemented with cancellous autologous contralateral iliac crest bone grafting was done (figure $5 \mathrm{c}$ and $5 \mathrm{~d}$ ). Patient recovery was uneventful and he was allowed protected weight bearing after six weeks of his second injury progressing to full weight bearing with support walking at three months. Radiological assessment at four months showed bony consolidation (figure 5e and 5f).

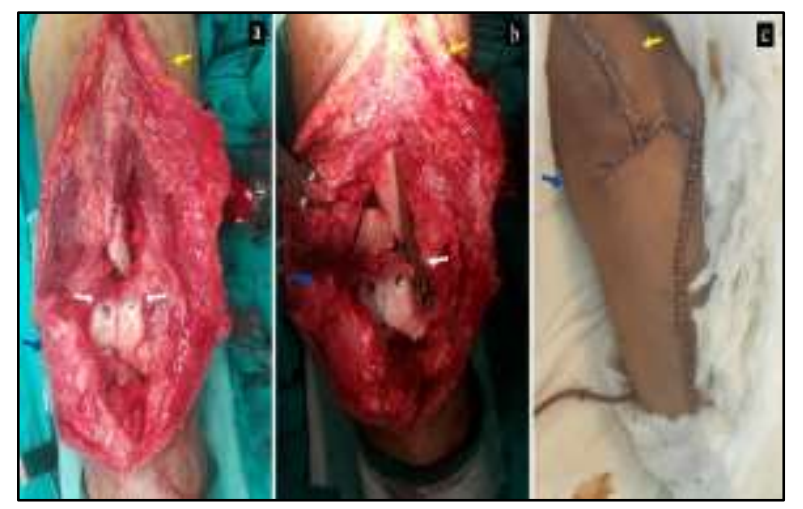

Figure 3: Intra operative photographs showing stabilization of Hoffa fracture fixation (3a) with cannulated cancellous screw (white arrow) bilaterally, distal femur bridging locked plate (white arrow)

fixation supplemented ( $3 \mathrm{~b})$, and wound closure over a negative suction drain (3c).

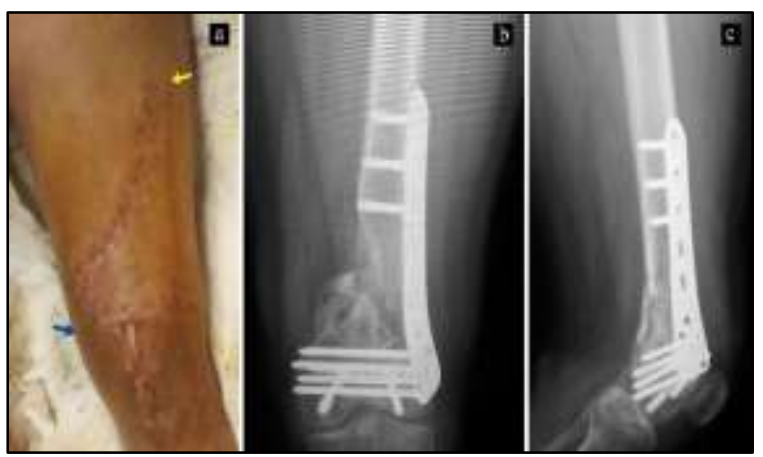

Figure 4: Photographs at 16 weeks of healed wound (4a), anteroposterior radiographs (4b) and lateral view radiograph $(4 \mathrm{c})$ shows good consolidation.

At the last follow-up at twenty months of his primary injury, the patient had a painless range of knee flexion to 130 degrees with no extensor lag or ligamentous laxity (figure $6 \mathrm{a}$ and $6 \mathrm{~b}$ ). He was ambulant bearing full weight without any support (figure $6 \mathrm{c}$ ). The patient gave a written informed consent for using his data for publication.

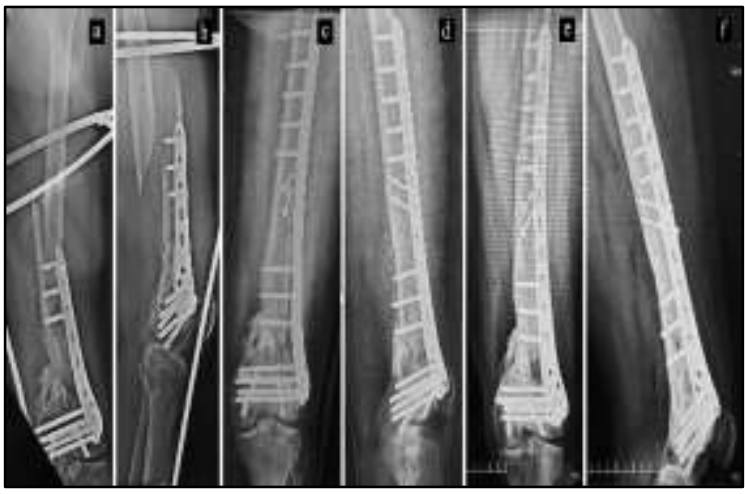

Figure 5: Peri implant femoral fracture plain preoperative anteroposterior (5a), lateral (5b) radiographs, and immediate post-operative plain anteroposterior $(5 \mathrm{c})$, lateral $(\mathbf{5 d})$ radiographs, and

follow up at 4 months anteroposterior (5e), lateral (5f) radiographs showed union without any avascular necrosis or implant loosening.

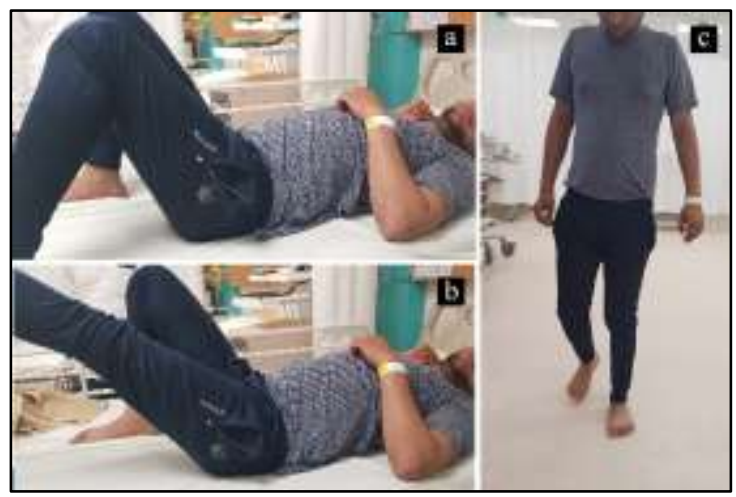

Figure 6: Clinical photograph at 20 weeks of primary injury with range of flexion upto130 $(6 a)$ and no extensor lag (6b) with painless full weight bearing left lower limb (6c).

\section{DISCUSSION}

Distal femoral fractures comprise approximately 4-6\% of all femoral fractures. ${ }^{2,12}$ Open supracondylar femur fractures are rare, complex injuries which occur in high energy polytrauma patients. ${ }^{12}$ They are complicated by bone loss, contamination, compromised soft tissues, and mishandled host condition with late presentations. ${ }^{2,13}$ Possibility of infection and non-union require meticulous treatment considerations. ${ }^{14}$ The Hoffa fractures remain apocalyptic injuries with high morbidity. ${ }^{5}$ The lateral condylar Hoffa fractures are more common, since the lateral femoral condyle has a greater anteroposterior dimension and the knee has a natural valgus orientation, leading to an earlier impingement against the tibial plateau than its medial counterpart. ${ }^{2,5,6}$

Diagnosis with both plain radiographs and Computed tomography (CT) may be useful..$^{2}$ The interpretation of radiographs can present difficulties, as in our case, because the Hoffa's fracture may be obscured in the anteroposterior 
projection by the intact anterior part of the condyle. ${ }^{6}$ Nork et al has suggested that up to $30 \%$ of Hoffa's fracture gets overlooked on plain radiographs and advocated the use of CT of intra-articular distal femoral fractures to assess the fracture pattern and plan the surgical fixation. ${ }^{5}$

Non-operative treatment or a late reconstructive approach of these complex injuries leads to malalignment, rotational deformities, loss of knee movement, joint contractures and subsequent arthritis. ${ }^{2,6,13}$

The goals of clinical management of distal femoral fractures is the anatomic reduction of the articular surface and restoration of limb length, rotation, and alignment. ${ }^{2,12}$ Limb reconstruction attempt in complex injuries with articular involvement and bone loss usually involves multiple surgeries which are time consuming, financially demanding, and often yield poor results. ${ }^{3}$

Advocates argue that in initial surgery one should do a thorough initial debridement, antibiotic bead placement for wound sterilization, and restoration of length and alignment with fixator or a lateral distal femur locking plate. Once patient achieves physiologic and mental wellness and the soft tissues have revascularized sufficiently, bone grafting is to be performed in addition to medial column plating to achieve rigid fixation and prevent the distal femur from Varus collapse. However, with nearly a year of life spent attempting to salvage their limb, this process proved taxing for most patients. Further, despite a united well-aligned limb, knee dysfunction precluded normality in most patients. ${ }^{13}$ Delayed single stage defect reconstruction with autologous, free, nonvascularized fibular grafting and fixation was advocated in treatment of significant bone loss due to acute, massive traumatic metaphyseal bone loss of the femur with acceptable short and long-term outcomes. ${ }^{3,15}$

For a compound injury presenting within a golden hour period available for management, we did a thorough debridement and reassessment of severity of compounding and better fracture evaluation at the earliest. A dilemma for use of external fixator, illizarov fixator or definitive fixation may be a debatable issue however, we considered a definitive fixation will allow better fracture stability and potential for biological soft tissue healing. Considering that we had loss of bony fragments, the defect was supplemented by primary bone grafting to give a better environment for union. An issue of creating an additional different site morbidity and an increased chance of infection may be another debate. However, pre-operative planning and discussion regarding possible challenges allowed a per operative decision making simple regarding whether to use an external fixator or definitive fixation, whether to use primary bone graft or allow secondary procedures later on.

Swash-buckler approach is commonly advocated for the direct complete exposure of comminuted intraarticular distal femur fractures, sparring of quadriceps muscle apparatus and facilitating any late arthroplasty procedures. ${ }^{16} \mathrm{~A}$ modified direct surgical approach was utilized by removal of sutures from loosely repaired wounds and re-assessment of soft tissue envelope. All small lacerations connected to obtain an elliptical wound which was extended distally up-to tibial tuberosity and proximally curved laterally to allow exposure of distal femoral shaft for plate fixation.

The standard method of treating Hoffa fractures has been lag screw fixation. ${ }^{7}$ Lag screws directed from posterior to anterior offer a mechanical advantage over screws placed from anterior to posterior. ${ }^{6}$ Partially threaded cancellous or headless screws of $6.5 \mathrm{~mm}$ or $4 \mathrm{~mm}$ in length supplemented by an anti-glide/buttress plate in osteoporotic bones or screws inserted just proximal to the Hoffa's fragment to buttress it against a shearing stress are the options the operating surgeon may avail. ${ }^{5}$ There is consensus yet to be reached on the method of fixation (in terms of anterior/posterior direction of screw insertion, and type/size/number of screws to use) that is both safe to perform and superior in providing interfragmentary compression. ${ }^{5}$ We believed that posterior buttress plate was not a viable option in our case. First, we would have had to extend our soft-tissue dissection significantly to gain access for plate positioning and fixation, thus compromising the blood supply to the fragments. Second, a plate of adequate length to act as a buttress plate would be required, compromising part of the posterior articular surface of the condyles.

Creating a solid construct with anatomic reduction and compressive fixation in accordance with the AO principles of fixation of intra-articular fractures is advocated. ${ }^{7}$ However, due to nature of the soft tissue injury in our case and associated comminuted metaphyseal distal femur fracture with loss of bony fragments, a biological fixation principle was used. Hoffa fracture fixation was supplemented by bridging of fracture with locked distal femoral plate for proper limb alignment, length and additional grafting procedure was done for defect augmentation. ${ }^{13}$

We followed a cautious approach during rehabilitation. Patient was allowed toe touch or non-weight bearing assisted mobilization for 6-12 weeks after surgery or until evidence of radiographic fracture healing.

Non-prosthetic peri-implant fracture (NPPIF) is a fracture in a bone with an existing non-prosthetic implant. It usually presented with low energy trauma in elderly osteoporotic group. A smaller subset of younger patients involved in high-velocity motor vehicle accidents occur infrequently. ${ }^{17}$ The management is challenged by previous surgery abnormal oriented scar, implant retention to implant revision, augmentation of bone healing potential and possibly avoiding infection to achieve a functional limb. ${ }^{17}$ 
Treatment options discussed in few reports include plate on plate osteosynthesis, re-osteosynthesis, supplementary intramedullary fixation or external fixator depending on fracture configuration, bone quality and soft tissue involvement. ${ }^{17,18}$ Peri-implant fractures present challenges in surgical management and a case-based approach should be followed with adherence to principles of fracture management to avoid failure. Evidence based recommendations are sparse..$^{17}$

Secondary arthritis may develop and need management. The patient should be counselled and explained in detail about the guarded prognosis and the possibility of late degenerative arthritis. ${ }^{5}$ Meticulous preoperative planning helped in avoiding any early complications. There was no evidence of avascular necrosis or failure of implant at the last follow up.

Open complex distal femur fracture presenting early will benefit from timely intervention and adherence to basic soft tissue management and bony reconstructions protocols. With advancement in trauma care, a single staged reconstruction allowed an early functional recovery with a favorable outcome. Secondary posttraumatic periimplant fracture complicated recovery in an already complex injury pattern. Complication rates for infection, non-union and implant failure are high in such cases. Surgical management strategies should be individualized with the aim to reduce complication and attain a good functional outcome.

\section{CONCLUSION}

Open complex distal femur fractures with associated articular involvement presented a unique management dilemma. A secondary peri-implant fracture added to the challenges. The treatment options should be wisely implemented. The early presentation within the golden hour period for an open complex distal femoral injury is an opportunity. A meticulous preoperative planning, intraoperative assessment to implement treatment modifications and postoperative early rehabilitation can lead to an early functional recovery in a difficult fracture pattern even with a secondary hit.

Funding: No funding sources Conflict of interest: None declared Ethical approval: Not required

\section{REFERENCES}

1. Forster MC, Komarsamy B, Davison JN. Distal femoral fractures: A review of fixation methods. Injury. Int J Care Injured. 2006;37(2):97-108.

2. Keudell AV, Shoji K, Nasr M, Lucas R, Dolan R, Weaver MJ. Treatment Options for Distal Femur Fractures. J Orthop Trauma. 2016;30:S25-7.

3. Iyengar R, Cherukuri N, Patnala C. Reconstruction of Traumatic, Open Supracondylar Femoral Fractures by Autologous Fibular Strut Grafting and CorticoCancellous Bone Grafting-A Single-Centre, Observational Study. J Orthop Traum. 2018;32(2):75-81.

4. Baruah JP, Bora S, Medhi H. The outcome following fixation of complex open distal femur fractures. Int J Orthop. 2019;5(4):297-301.

5. Kamal B, Krishna MA, Krishnan V, Kumar V, Goni $\mathrm{V}$, Rawall $\mathrm{S}$ et al. A rare case of bicondylar Hoffa fracture associated with ipsilateral tibial spine avulsion and extensor mechanism disruption. Chin J Traumatol. 2011;14(4):253-6.

6. Neogi DS, Singh S, Yadav CS, Khan SA. Bicondylar Hoffa fracture? A rarely occurring, commonly missed injury. Injury Extra. 2008;39(9):296-8.

7. Giotikas D, Nabergoj M, Krkovic M. Surgical management of complex intra-articular distal femoral and bicondylar Hoffa fracture. Ann R Colleg Surg Eng. 2016;98:e168-70.

8. Calmet J, Mellado JM, Forcada IG, Giné J. Open bicondylar Hoffa fracture associated with extensor mechanism injury. J Orthop Trauma. 2004;18(5):323-5.

9. Park KC, Lim SJ, Song YS, Hwang KT. Factors affecting peri-implant fracture following locking plate for osteoporotic distal femur fractures. Orthop Traumatol Surg Res. 2017;103(8):1201-4.

10. Gustilo RB, Mendoza RM, Williams DN. Problems in the management of type III (severe) open fractures: a new classification of type III open fractures. J Trauma. 1984;24:742-6.

11. Mu“ller ME, Nazarian S, Koch P. The AOClassification of Fractures. Berlin:Springer. 1990.

12. Rajagopal HP, Mohan M, Pilar A, Tamboowalla KB. Reconstruction of complex distal femur open fracture with distal femoral locking plate and primary autologous free fibular graft. Int $\mathrm{J}$ Res Orthop. 2017;3:1066-9.

13. Dugan TR, Hubert MG, Siska PA, Pape HC, Tarkin IS. Open supracondylar femur fractures with bone loss in the polytraumatized patient - Timing is everything! Injury. Int J Car Injured. 2013;44:182631.

14. Thakkar CV, Dwivedi MS. Open distal femur fractures: Treatment Principles. Int J Orthop Sci. 2018;4:732-6.

15. Gawri V, Garg Y, Singh D, Garg RS, Choudhary N. Complex open distal femur fracture managed by primary autogenous fibular graft in conjunction with DFLP-A case report. J Clin Diag Res. 2015;9(11):RD01.

16. Starr AJ, Jones AL, Reinert CM. The" swashbuckler": a modified anterior approach for fractures of the distal femur. J Orthop Trauma. 1999;13(2):138-40.

17. Chan LWM, Gardner AW, Wong MK, Chua K, Kwek EBK. Non-prosthetic peri-implant fractures: classification, management and outcomes. Arch Orthop Trauma Surg. 2018;138(6):791-802. 
18. Ma CH, Chiu YC, Wu CH, Tsai KL, Wen TK, Jou IM, Tu YK. Plate-on-plate technique for treating peri-implant fractures of distal femoral locking plate: a retrospective study of 11 patients. Arch Orthop Trauma Surg. 2019;139(9):1245-51.
Cite this article as: Tomar L, Govil G, Dhawan P. Open complex distal femur intraarticular fracture with Hoffa extension, extensor mechanism tear and post-recovery secondary traumatic peri implant fracture: report on an uncommon chronology. Int $\mathrm{J}$ Res Orthop 2020;6:1335-40. 